\title{
Demonstration of flywheel-based travelling-wave thermoacoustic engine
}

\author{
Takumaru Watanabe ${ }^{1, *}$,Eita Shoji ${ }^{1}$, Tetsushi Biwa ${ }^{1}$, and Guillaume Penelet $^{2}$ \\ ${ }^{1}$ Department of Mechanical Systems Design, Tohoku University \\ Aramaki, Aoba-ku, Sendai, 980-8579, Japan \\ ${ }^{2}$ Laboratoire d'Acoustique de l'Université du Mans \\ Avenue Olivier Messiaen, 72085 Le Mans Cedex 9, France
}

\begin{abstract}
We report test results on a thermoacoustic engine having a piston-crank-flywheel assembly. The engine maintained steady rotation states when the heating temperature was increased more than $114^{\circ} \mathrm{C}$. The rotation frequency increased with the higher heating temperature and reached $14.5 \mathrm{~Hz}$ when it was $296^{\circ} \mathrm{C}$. The pressure versus volume diagram was created to deduce the power delivered to the piston-crank-flywheel assembly from measurements of the pressure of the working gas and the rotation angle of the flywheel.
\end{abstract}

\section{Introduction}

A thermoacoustic engine with a piston-flywheel assembly has been developed [1]. The thermoacoustic engine is the acoustical heat engine that is based on thermally induced oscillations of a gas column [2]. The output power of the acoustical engine is the acoustic power associated with the generated sound waves. So far, the attempts have been made to convert the output acoustic power to the electricity using a linear alternator $[3,4]$. The thermoacoustic engine with a flywheel has replaced the linear alternator with a flywheel that can be connected to a rotational alternator in the same way as a bidirectional turbine [5]. This flywheel rotation frequency increases with increasing the heating temperature, in contrast to the linear alternator based thermoacoustic engine that keeps the oscillation frequency while increasing the amplitude with the heating temperature.

A numerical simulation model has been proposed for the thermoacoustic engine with a flywheel, and the simulation results have been compared to the experimental results [6]. It was shown that the proposed numerical model captured the dynamics of the system quantitatively. The model incorporates the input acoustic impedance of the engine subsystem described by the thermoacoustic theory in the frequency domain and transforms it to the ordinary differential equation in the time domain, to couple it with the equation of motion of the flywheel. This calculation method needs further investigation to establish its validity. In this study, we report measurement results of the rotation angle and pressure during the steady operation of the engine. ${ }^{*}$

\footnotetext{
* Corresponding author: takumaru.watanabe.t6@dc.tohoku.ac.jp
} 


\section{Experiment}

Figure 1 shows a schematic illustration of the flywheel based thermoacoustic engine. The working gas of the engine was air at ambient temperature and pressure. The engine consisted of two subsystems: a looped tube and a piston-flywheel assembly.

The looped tube is called an engine subsystem made of a $40-\mathrm{mm}$ main tube and a $12-\mathrm{mm}$ feedback tube. The average length of the loop was $672 \mathrm{~mm}$. The main tube contained a regenerator and a pair of heat exchangers. The 35-mm long regenerator was made by stacking stainless steel mesh screens (\#30, wire diameter was $0.18 \mathrm{~mm}$ ). The heat exchangers were parallel plates with spacing of $1 \mathrm{~mm}$. The plate thickness was $0.5 \mathrm{~mm}$, and the length was 15 $\mathrm{mm}$. The cold heat exchanger was cooled by a circulating water at room temperature. The hot heat exchanger was heated by feeding a dc electrical current to an electrical heater wound around the heat exchanger body. The heating temperature $T_{\mathrm{H}}$ was monitored by using a sheathed thermocouple that was inserted into the boundary between the regenerator and the hot heat exchanger plate on the central axis of the main tube. At the bottom of the loop, the secondary cold heat exchanger was placed, which had no parallel plates inside.

Glass piston and cylinder (Tsubasa industry) were employed. The diameter of the piton was $35 \mathrm{~mm}$, and the length was $158 \mathrm{~mm}$. The position of the piston top dead center was adjusted to coincide with the top of the cylinder. An aluminum connecting rod connected the piston to a crank pin fixed on the flywheel that was a cylindrical brass plate with $7 \mathrm{~mm}$ thickness and $35 \mathrm{~mm}$ radius. The mass was totally $0.174 \mathrm{~kg}$ for the glass piston and the connecting rod. The mass moment of inertia of the flywheel plate was $1.44 \times 10^{-4} \mathrm{~kg} \mathrm{~m}^{2}$. The peak-to-peak stroke of the piston was adjusted to either $2 r=20,40$, or $60 \mathrm{~mm}$ by changing the crank pin position on the flywheel plate.

We measured the pressure of the working gas using a pressure transducer (DD104, JTEKT) mounted on a T-shaped part of the engine subsystem, and the rotation angle of the flywheel plate using a rotary encoder (MG-30, Microtech laboratory inc.) that consisted of a circular disk having evenly spaced 360 slits and an optical system detecting light pulses passing through the slits. The optical pulse signal was converted to the analogue voltage proportional to the angle of the disk by a counter (CNT-723, Coco Research inc.). The time intervals of the pulses were transformed to the analogue voltage proportional to the angular frequency by an F-V converter (KAZ-Mighty, Coco Research inc.). The analogue signals of the pressure, angle, and angular frequency were recorded with a multichannel A/D converter (DS-2000, Onosokki inc.). The measurements were conducted when the engine was in the steady rotation states that was achieved after initial rotation by hand. 
(a)

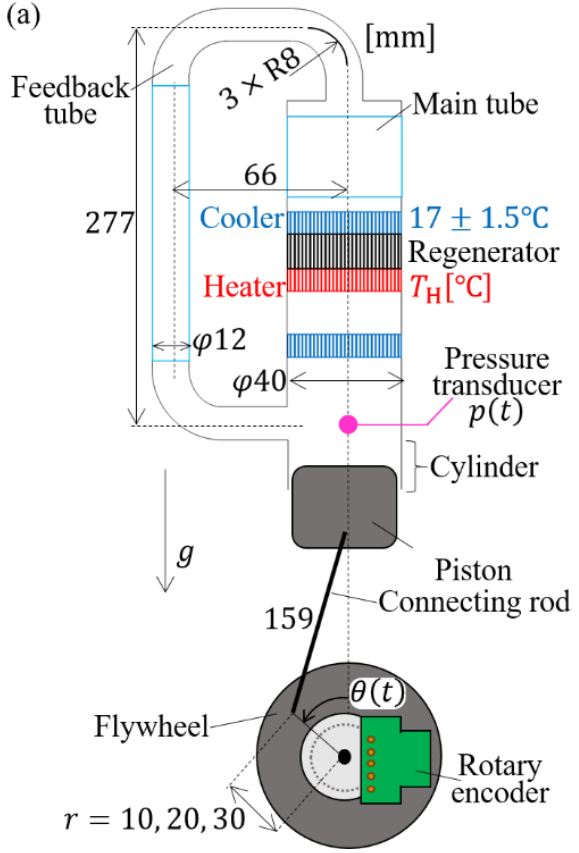

(b)

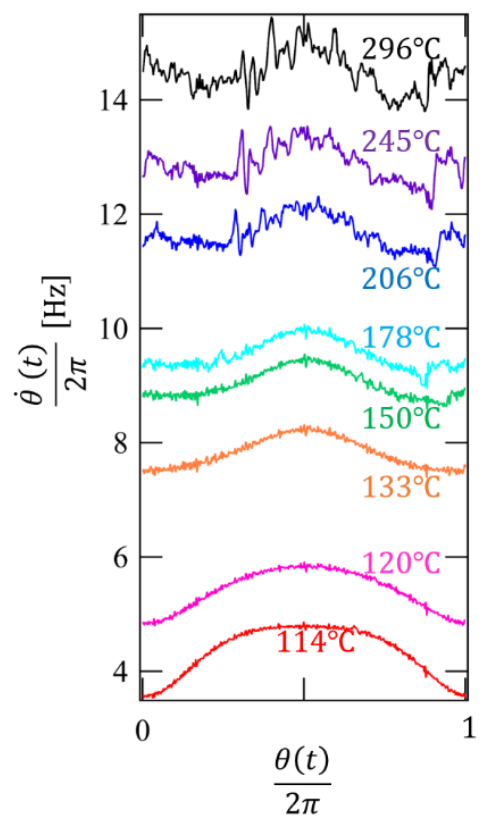

Figure 1. (a) schematic illustration of the present thermoacoustic engine with a flywheel (not in scale). $g$ stands for the gravitational acceleration. (b) Relation between angular frequency $\dot{\theta} /(2 \pi)$ and angle $\theta$ when the crank radius was set to $r=10 \mathrm{~mm}$. Colors represent the heating temperature $T_{\mathrm{H}}$.

\section{Results and discussion}

Figure 1(b) shows the relation between the angular frequency $\dot{\theta} /(2 \pi)$ and angle $\theta$ when the crank radius was set to $r=10 \mathrm{~mm}$. Colors represent heating temperature $T_{\mathrm{H}}$. With the lowest temperature $T_{\mathrm{H}}=114^{\circ} \mathrm{C}$, the $\dot{\theta} /(2 \pi)-\theta$ relation showed a broaden peak, which is qualitatively consistent with the calculation result in the previous report [6]. This result reflects the impact of gravitational acceleration, as the engine was set vertically. The rotation frequency was as low as $4.0 \mathrm{~Hz}$ in average, but it was elevated as $T_{\mathrm{H}}$ was increased. With $T_{\mathrm{H}}$ $=296^{\circ} \mathrm{C}$, the rotation frequency was increased to $14.5 \mathrm{~Hz}$. The shape of the $\dot{\theta} /(2 \pi)-\theta$ relation also changed to have additional peak near $\theta=0$. We consider that the thermal properties of the engine subsystem should be considered to explain this result, in addition to the rotation dynamics of the piston-crank-flywheel assembly.

Figure 2 shows a relation of the temporal average of the angular frequency and the heating temperature when the crank radius was changed. As can be seen in Fig. 2, the engine entered the steady rotation state with $T_{\mathrm{H}}=101^{\circ} \mathrm{C}$ when the crank radius was $r=20 \mathrm{~mm}$. When $T_{\mathrm{H}}$ was increased to $212^{\circ} \mathrm{C}$, the angular frequency reached $13.7 \mathrm{~Hz}$. When the crank radius was $30 \mathrm{~mm}$, the angular frequency was almost the same as that of $20 \mathrm{~mm}$. We will test the engine by increasing the crank radius to see the engine performance. 


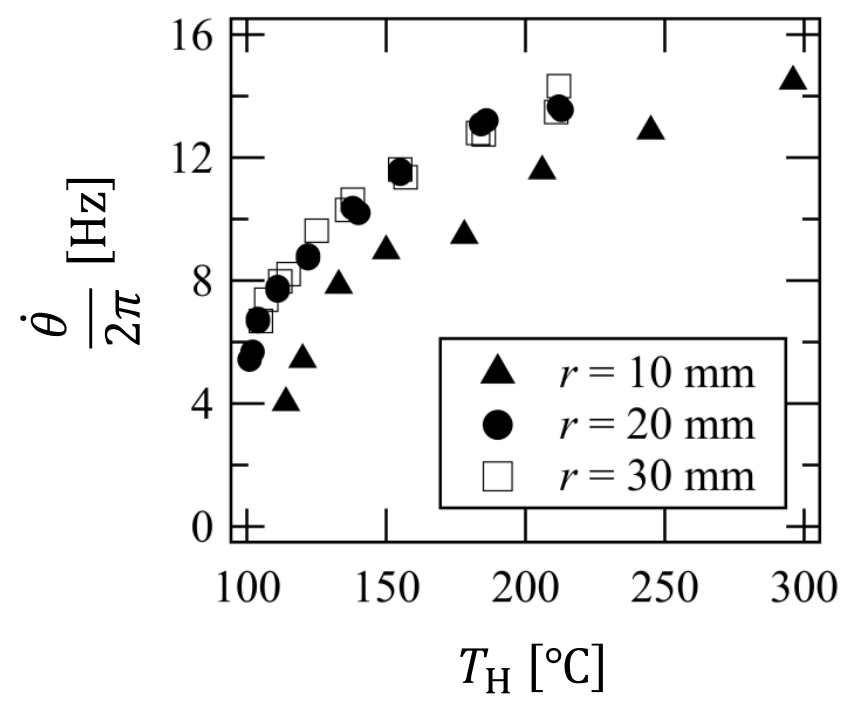

Figure 2. Time-averaged rotation frequency as a function of heating temperature $T_{\mathrm{H}}$. Markers represent crank radius.

It should be noted that the steady rotation of the flywheel was sustained by the acoustic power created in the regenerator. As demonstrated in the previous report [1], the pressure of the working gas oscillates almost in phase with the velocity in this engine. Therefore, the gas thermodynamic cycle in the regenerator region is essentially the same as in the conventional mechanical Stirling engine. A pulse-tube engine has a single piston engine, but the energy conversion mechanism intrinsically relies on irreversible processes in the stack region [7]. The present engine is classified into the Stirling engine as it relies on the reversible thermodynamic processes in the regenerator region.

Shown in Fig. 3 is a pressure $P$ versus volume $V$ diagram when the crank radius was $r=$ $10 \mathrm{~mm}$ and heating temperature was $T_{\mathrm{H}}=114^{\circ} \mathrm{C}$. The pressure $P$ stands for the excess pressure from the mean pressure that is the atmospheric pressure, whereas the volume $V$ means the swept volume of the piston that was calculated from the measured angle $\theta$ by

$$
V=r A(1-\cos \theta)
$$

where $A$ is the cross-sectional area of the cylinder. The $P-V$ diagram drew a tilted closed orbit rotating in a clockwise direction. The enclosed area, which was turned out to be 70.1 $\mathrm{mW}$, corresponds to the power delivered from the engine subsystem to the piston-cylinderflywheel assembly. In other words, it is the power dissipated in the assembly because the present engine does not have a load.

From Fig. 2, it is found that the rotation frequency increases with heating temperature $T_{\mathrm{H}}$. It also increases with the larger crank radius. Therefore, the power delivered to the assembly should be the largest when the crank radius was adjusted to $r=30 \mathrm{~mm}$ and the heating temperature was raised to $T_{\mathrm{H}}=212^{\circ} \mathrm{C}$. We are planning to put some load to extract a shaft power from the engine.

The $P-V$ diagram should reflect the thermal properties of the engine subsystem that contained the regenerator and heat exchangers. The input acoustic impedance of the engine subsystem can be theoretically and numerically obtained by using the thermoacoustic theory. In the future study we will analyze the relation of the $P-V$ diagram with the input acoustic impedance of the engine subsystem. 


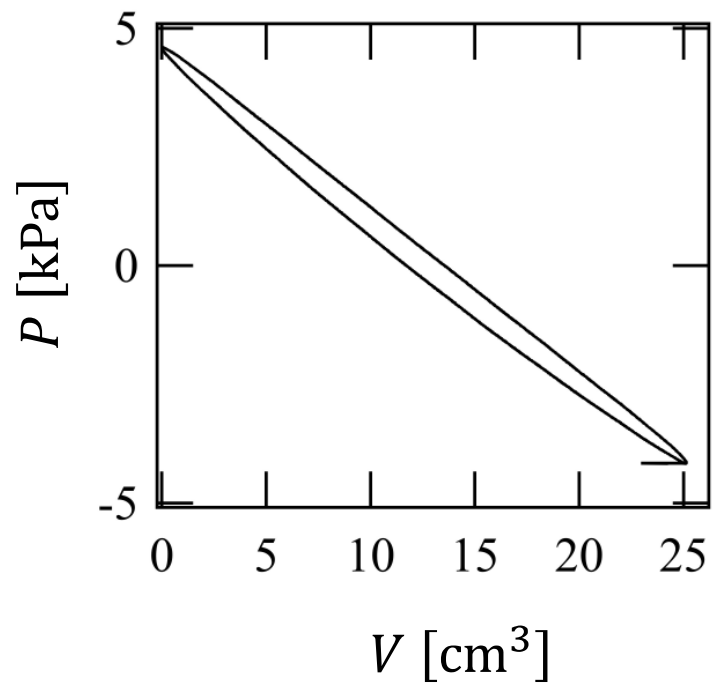

Figure 3. Pressure versus volume diagram measured when $T_{\mathrm{H}}=114^{\circ} \mathrm{C}$ and $r=10 \mathrm{~mm}$.

\section{Summary}

We have tested the thermoacoustic engine with the piston-crank-flywheel assembly. The engine entered the steady rotation state when the heating temperature was increased more than $114^{\circ} \mathrm{C}$. The rotation frequency reached $14.5 \mathrm{~Hz}$ when the heating temperature was $296^{\circ} \mathrm{C}$. The pressure versus volume diagram was created to deduce the output power from the engine subsystem. The future work will address the physics behind the $P-V$ diagram and the rotation frequency from a thermoacoustical point of view.

\section{References}

[1] T. Biwa, T. Watanabe, G. Penelet, Appl. Phys. Lett. 117, 243902 (2020).

[2] T. Yazaki, A. Iwata, T. Maekawa, and A. Tominaga, Phys. Rev. Lett. 81, 3128 (1998).

[3] S. Backhaus, E. Tward, and M. Petach, Appl. Phys. Lett. 85, 1085-1087 (2004).

[4] T. Bi, Z. Wu, L. Zhang, G. Yu, E. Luo, and W. Dai, Appl. Energy 185, 1355-1361 (2017).

[5] M. A. G. Timmer, K. de Blok, and T. H. van der Meer, J. Acoust. Soc. Am. 143, 841-857 (2018).

[6] G. Penelet, T. Watanabe, T. Biwa, J. Acoust. Soc. Am. 149, 1674 (2021).

[7] T. Yoshida, T. Yazaki, H. Futaki, K. Hamaguchi, T. Biwa, Appl. Phys. Lett. 95, 044101 (2009). 